\title{
Efficacy and Safety of Percutaneous Nephrolithotomy in Patients Over 70 Years with Kidney Stones
}

\author{
Todea-Moga Ciprian, Boja Radu, Porav-Hodade Daniel, Maier Adrian, Ghirca Veronica, Mártha Orsolya \\ University of Medicine and Pharmacy Tirgu Mures, Romania, Clinic of Urology
}

Introduction and objectives: Percutaneous nephrolithotomy represent the main indication for patients with kidney stones, even in the presence of various comorbidities. In our clinic open surgery for this pathology is less than $0.5 \%$ of allprocedures for renal stones. The objective of this paper is to assess the safety and efficacy of this procedure in patients over 70 years.

Material and methods: A retrospective study was performed for a period of 16 years (1997-2012). A totally of 323 patients entered in this study (162 women, 161 men), aged over 70 and with renal stones They were treated endoscopically by percutaneous nephrolithtomy or anterograde ureteroscopy. 85 patients $(26.31 \%)$ had comorbidities that were preoperatively diagnosed and treated where necessary.

Results: Overall status of "stone free" at the end of surgery was present in 263 patients

(81.42\%). 60 patients (18.58\%) had residual fragments. Residual stones were solved by a new percutaneuos nephrolithtomy session, spontaneous elimination or extracorporeal shock wave lithotripsy. The most common complications were bleeding and infection. We had no deaths. No hemostasis nephrectomy was necessary.

Conclusions: Recognized preoperative comorbidities do not represent risk factors in elderly patients, but it requires a rigorous evaluation in the preoperative period. The number, size and complexity of stones directly influences the state "stone free" at the end of surgery.

Keywords: renal stones, percutaneuos nephrolithtomy, elderly patients

Received: 23 September 2015 / Accepted: 28 November 2015

\section{Introduction}

The treatment of kidney stones in the elderly patients is an actual problem due to the aging of the population. The incidence of the renal stones in elderly population is above $10 \%$ and the male/female ratio is greater than $2[1-3]$.

The therapy of lithiasis pathology experienced a change of strategies by the appearance of endoscopic techniques. The majority of small stones can be eliminated spontaneously, others are treated by extracorporeal lithotripsy (ESWL) [4]. For those that can not be solved, the endoscopic techniques are the solution, the open technique is applied only exceptionally. The laparoscopic techniques and especially flexible ureteroscopy reduce the number of percutaneous nephrolitotomy (PCNL). However PCNL therapy remains the gold standard techniques for kidney stones. Even if the risks of anesthesia for PCNL are higher in elderly patients over 70 years, percutaneous nephrolithotomy is still needed for complex or not complicated urolithiasis [5].

In our clinic the open surgery for this condition represent less than $0.5 \%$ of all surgeries for kidney stones.

\section{Material and methods}

We performed a retrospective study over a period of 16 years (1997-2012) that included a total of 323 patients (162 women, $161 \mathrm{men}$ ), aged over 70 years, with renouretheral stones. Staghorn stones were found in a total of

${ }^{*}$ Correspondence to: CiprianTodea-Moga

E-mail: ciprian.todea@gmail.com
55 patients. Uretheral stones were found in 38 patients and were resolved after push back.

Preoperative antibiotic treatment was indicated in patients who presented positive urine cultures (83 patients). In this group of patients we administrated prophylactic preoperative antibiotics for $24-48 \mathrm{~h}$. For all patients was performed spinal anesthesia and stone extraction was performed by nephroscopic percutaneous approach. We performed ultrasonic or pneumatic fragmentation followed by fragments extraction trought the nephroscope sheath or Amplatz sheath for larger fragments.

We performed internal uretheral drainage for patients with uretheral stones, but also for patients who presented a lumbar fistula more than 72 hours after the suppression of the nephrostomia. Uretheral drainage was maintained between 4 and 6 weeks.

Postoperative ultrasound examination was performed in all patients within 48 hours after surgery before nephrostomy suppression. Percutaneous nephrostomy was suppressed at 48 hours, except in cases when debloating of nephrostomy tube was followed by bleeding at nephrostomy level (24 patients). In these cases nephrostomy extraction was performed between 48 and 96 hours postoperatively. Follow up was performed at 3 months postoperative. This consisted of abdominal ultrasound, urine examination, renal radiography.

A patient was considered to be cured if the control of three months showed no residual fragments ("stone free") and analysis of the urine was normal. 


\section{Results}

A total of 263 patients $(81.42 \%)$ were considered "stone free" at the end of treatment. The "stone free" was not influenced by patient age, but it was influenced by the volume and complexity of the stone. The only comorbidity that represented technical problems was the bilateral hip arthrosis. We did not notice any influence of the this comorbidity on the outcome of "stone free" in these patients.

A total of 13 patients have urinary fistulae after nephrostomy extraction. In 3 patients the urinary fistulae was resolved spontaneously, but for 10 patients we inserted internal uretheral drainage. The most important complications were represented by fever and bleeding.

The urinary infection, manifested by febrile climbing was seen in 170 patients $(52.63 \%)$. Of these 47 had positive urine cultures before the endoscopic intervention. The most common bacteria in preoperative urine cultures were Escherichia coli (28.11\%) and Pseudomonas (21.13\%). There was no correlation between febrile episodes of patients and "stone free" status after surgery, or performed at 3 months.

Postoperatively, 78 patients were infected (24.14\%). In our experience postoperative urinary infection is closely related to the early suppression of the nephrostomy tube, the number of nephrostomy tracks and number of operatory sessions. No sepsis status was found.

Bleeding was seen in 22 patients $(6.81 \%)$. Out of this in 20 patients the bleeding appeared during or immediately after the surgery and in 2 cases at 48 hours after the removal of nephrostomy. Bleeding was solved with the nephrostomy balloon, blood transfusions and plasma. We did not performe hemostatic nephrectomy in this group of patients. No deaths were registered.

\section{Discussion}

Percutaneous nephrolithotomy for renal stones over $2 \mathrm{~cm}$ diameter remains a "gold standard" technique. For unique stones under $2 \mathrm{~cm}$ ESWL or flexible ureteroscopy is the first-line therapy $[1,6]$. Preexisting comorbidities in patients over 70 years had no influence on operative techniques, postoperative outcomes or complications in the conditions in which they were recogniyzed and treated preoperatively. There were no cases of contraindication of the surgical therapy because of these comorbidities. In the presence of comorbidities, old age, the success of the intervention in this group of patients is directly related to the operative time. For this reason, unique stones were extracted through nephroscope or 28 and $30 \mathrm{Ch} \mathrm{Amplatz}$ sheath, processes that increase the rate of "stone-free" status in these patients $[7,8]$. Besides reducing operative time, the Amplatz sheaths reduce the risk irrigation fluid absorption (decreased pressure) and bleeding. Sheath's diameter is considered by some authors as an additional factor that may influence the bleeding, but does not influence the appropriate parenchymal distruction $[9,10]$. In our study, usage 28 or $30 \mathrm{Ch}$ Amplatz sheaths did not increase the incidence of bleeding in elderly patients, with no influence in renal function [11].

In our observation, the duration of nephrostomy drainage tube directly influences the incidence of postoperative urinary infection. This influence directly the number of hospitalization days after surgery. A longer maintenance of this tube is indicated in patients with residual fragments that must be extracted in another surgical session by this preexisting path or, if not possible, by creating a new nephrostomy path. In this case the solution of ESWL sessions for the remaining fragment is welcomed. The rate of "stone free" at the end of surgery was $81.42 \%$. The percentage of patients "stone free" after PCNL is certainly influenced by the existence of a single initial stone and the possibility to extract it intact by Amplatz sheath, or directly through the nephrostomy paths.

\section{Conclusions}

1. Present comorbidities in patients over 70 years is not a particular risk factor for percutaneous nephrolithotomy, if this conditions are recognized and treated preoperatively.

2. Associated comorbidities not alter the rate of "stone free" post PCNL.

3. The number, size and complexity of the stones directly influence the "stone free" status at the end of the intervention.

4. Tehnically the PCNL in elderly patients is not a complicated intervention and it can be repeated without particular risks, in relation to the biological status of the patient.

5. The risk of bleeding or infection do not increase in patients over 70 years.

\section{Acknowlegment}

This paper was published under the frame of European Social Found, Human Resources Development Operational Programme 2007-2013, project no. POSDRU/159/1.5/S/133377.

\section{Conflicts of interest}

The authors report no conflicts of interest.

\section{References}

1. Kamphuis GM, Baard J, de la Rosette JJ, et al. Lessons learned from the CROES percutaneous nephrolithotomy global study. World J Urol, 2015;33:223-233.

2. Sahin A, Atsu N, Ersem E, et al. Percutaneous nephrolithotomy in patients aged 60 years or older. J Endourol. 2001;15:489-491.

3. Reynard J, Brewster S, Biers S. Oxford Handbook of Urology, 3rd ed. Oxford University Press, 2013;428-460.

4. Sheir KZ, Cad HM. Prospective study of the effects of shock wave lithotripsy on renal function: role of postshock wave lithotripsy obstruction. Urology, 2003;61:1102-1106.

5. Anagnostou T, Thompson T. Safety and outcome of percutaneous nephrolithotomy in the elderly: retrospective comparison to a younger patient group. J Endourol. 2008;22:2139-2145.

6. Segura JW, Preminger GM, Assimos DG, et al. Nephrolithiasis clinical guidelines panel summary report on the management of staghorn calculi. J Urol. 2010;151:1648-1651. 
7. BojaR. Chirurgia percutanata reno-ureterala. Ed. Leda\&Muntenia, Constanta, 2000:77-78.

8. Mullholland CK, Kernohan RM. Splitting the Amplatz sheath to facilitate percutaneous stone extractions. Br J Urol. 1994,74:375-376.

9. Karami H, Mohsen M, Golshan A, et al. Does age affect outcomes of percutaneous nephrolithotomy? J Urol. 2010;7:17-21.
10. Belabay MD, Varogh E, Devrine $H$, et al. Qualitative evolution of renal parenchymal mass with 99m technetium dimercapto-succinic acid scintigraphy after nephrolothotomy. J Urol. 1997;157:1226-1228.

11. Kukreja RA, Desai MR, Sabnis RB, Patel SH. Fluid absorbtion during percutaneous nephrolithotomy: does it matter? J Endourol. 2002;16:221 224. 